\title{
A novel mutation in the CTSC gene in Iranian family with Papillon-Lefevre Syndrome
}

\author{
Mahmoud Ghanei ${ }^{1}$, Mohammad Abbaszadegan ${ }^{1}$, Mohamad Mahdi Forghanifard ${ }^{2}$, Azadeh \\ A'rabi $^{1}$, and Hamid Reza Arab ${ }^{1}$ \\ ${ }^{1}$ Mashhad University of Medical Sciences \\ ${ }^{2}$ Islamic Azad University Damghan Branch
}

May 5, 2020

\begin{abstract}
Background: PLS is a rare autosomal recessive disorder characterized by the palmar-plantar hyperkeratosis and severe degeneration of the periodontium. The defects in cathepsin $\mathrm{C}$ gene, are responsible for PLS. In this study, we analyzed the whole exomes of CTSC gene in a family with history of PLS. Methods: Genomic DNA was extracted from peripheral blood and genotype analysis was performed. The mutated protein sequence was used to find the best possible tertiary structure for homology modeling. The homology modeling of the novel mutation was then performed using the online Swiss-Prot server. The results were also analyzed for to verify its validity. Results: The analysis of CTSC gene elucidated a novel insertion GAC. The novel mutation was proved by analyzing 50 healthy control volunteers. Modeling of the novel found mutation in CTSC gene revealed structural defects that may have caused the functional abnormalities. Discussion: The structural analysis of the mutated protein model identifies changes in the stereo-chemical and the energy level of the mutated protein. Since this protein play a role in the activation of granule serine proteases from cytotoxic $\mathrm{T}$ lymphocytes, natural killer cells, mast cells, such structural defects may lead to its malfunction causing dysfunctioning of immune defense mechanisms.
\end{abstract}

\section{Introduction}

Papillon-Lefevre syndrome (PLS; OMIM245000) is a rare inherited autosomal recessive disorder firstly reported by French physicians Papillon and Lefevre in 1924. According to the latest reports, the prevalence of this syndrome is 1-4 in a million people with equal distribution in males and females(Cury et al., 2002; R J Gorlin, Sedano, \& Anderson, 1964; Basapogu Sreeramulu, Shyam, Ajay, \& Suman, 2015). Although, a variety of studies has recently reported different affected Iranian families with PLS, there is not any accurate incidence of PLS in Iranian families(Arnold, Bordoli, Kopp, \& Schwede, 2006; Farjadian, Kiyanimanesh, Abbaszadegan, \& Lotfazar, 2007; Farjadian, Lotfazar, \& Ghaderi, 2008; Moghaddasian et al., 2014). The clinical symptoms of the syndrome is normally revealed in the first four years of patient life (It may be already present in the first 3 months of life however generally the palmoplantar hyperkeratosis and the severe periodontitis present simultaneously between 1 and 4 ages)(Farkas et al., 2013; Papillon, 1924; B Sreeramulu, Haragopal, Shalini, Sudha, \& Kiran, 2012). This disorder is characterized by a symmetrical palmar-plantar hyperkeratosis - dry scaly patches on the palms and the soles's skin- and severe inflammation and degeneration of the structures surrounding and supporting the teeth (periodontium)(Robert J Gorlin, 2000). Usually, complete deciduous tooth loss occurs before the 6 years of birth and then, the periodontitis disappears until all permanent teeth are fully grown out. All permanent teeth are normally lost when the patients turn 15 years old(Robert J Gorlin, Cohen Jr, \& Hennekam, 2001; Wu et al., 2016).

Other symptoms of PLS are hyperhidrosis, intracranial calcification, arachnodactyly, increased susceptibility 
to infections, hearing loss, mental retardation, oculocutaneous albinism, bent nail syndrome, corneal cell hyperkeratosis, acanthosis nigricans, thicker cutaneous granular layer, etc(Almuneef, Al Khenaizan, Al Ajaji, \& Al-Anazi, 2003; Borroni et al., 1985; Farkas et al., 2013; Haneke, 1979; Khandpur \& Reddy, 2001; Oğuzkurt, Tanyel, Büyükpamukçu, \& Hiçsönmez, 1996; Basapogu Sreeramulu et al., 2015; Tosti, Manuzzi, Bardazzi, \& Costa, 1988; Wani, Devkar, Patole, \& Shouche, 2006; Wu et al., 2016). Furthermore, this disorder may also be accompanied with other symptoms such as the skin, liver, kidneys and brain abscesses(R J Gorlin et al., 1964; Kanthimathinathan et al., 2013; Mercy, Singh, Ghorpade, Das, \& Upadhyay, 2013; Morgan, Hannon, \& Lakhoo, 2011).

Susceptibility to bacterial infections (often in respiratory tract) is approximately increased in $20-25 \%$ of patients with recurrent pyogenic infections as a result of decreased neutrophil, lymphocyte or monocyte functions in PLS patients $(6,12)$. The quality of patient's life can be improved by medications for hyperkeratosis and periodontal care. However, periodontal management of PLS is still a big challenge for treatment(Lundgren, Crossner, Twetman, \& Ullbro, 1996; Lundgren \& Renvert, 2004; Tekin et al., 2016).

The cathepsin C (CTSC) gene is mapped on chromosome 11q14.2 and encodes a cysteine-lysosomal protease called cathepsin C. Mutations in this gene, are responsible for Papillon-Lefevre syndrome(Hart et al., 1999; Toomes et al., 1999). Now, 91 CTSC mutations have been reported in PLS patients(http://www.hgmd.cf.ac.uk/ac/gene.php?gene $=C T S C$, n.d.; Pallos et al., 2010). Approximately more than half of these mutations are missense; however, a broad spectrum of mutation types have been described(Tekin et al., 2016).

Recently it has been revealed that Loss of function in lysosomal protease cathepsin $\mathrm{C}$ is the main genetic cause of the disease. This tetrameric protein plays a critical role in the removal of dipeptides from free $\mathrm{N}$-terminal of some hormones (including gastrin, glucagon and angiotensin II) and the activation of granule serine proteases from cytotoxic T lymphocytes, natural killer cells (granzymes A and B), mast cells (tryptase and chymase) and neutrophils (cathepsin G and elastase)(de Haar et al., 2004; Idon, Olasoji, \& Fusami, 2015; Romero-Quintana et al., 2013; Toomes et al., 1999).

Since 1999, several mutations have been reported in the CTSC gene majorly related to PLS. Herein, we present the case of a 6-year-old boy affected by insertion GAC (p.122ins.T) in the third exon of the CTSC gene.

Material and Methods

\section{Patients:}

This study was performed on a PLS patient and his parents. They exhibited some of common symptoms of PLS such as palmar-plantar hyperkeratosis, periodontitis, etc. The declared consent form was obtained from all members of the family. The study was approved by the Ethics Committee of Mashhad University of Medical Sciences (\# 910523). The clinicopathological data of patient is summarized in Table 1.

\section{Genotype analysis:}

Genomic DNA was extracted from peripheral blood leukocytes of the proband as well as his parents. Genotype analysis was performed on extracted DNA using specific primer sets for all seven exons of CTSC gene(Moghaddasian et al., 2014). Both strands of PCR products were directly sequenced using ABI Prism 310 Automated Sequencer and analyzed with the DNA sequence assembly software (Sequencher 4.10.1, Gene Codes Corporation). Numbering of CTSC nucleotides and amino acids was in accordance with the reference sequence on Gene Bank (NM_001814). Fifty healthy volunteers were also analyzed as a control group.

\section{Structural Analysis and protein modeling:}

To model the newly identified mutation (p.122insT), the mutated CTSC amino acid sequence was compared to template databases using the online server Swiss-Prot Template Identification tool(Arnold et al., 2006; Biasini et al., 2014; Kiefer, Arnold, Künzli, Bordoli, \& Schwede, 2008) on http://swissmodel.expasy.org. 
The most similar template to the target mutated protein, with $100 \%$ sequence identity, was human cathepsin C, which has been solved to $2.15 \AA$ K KBA including the amino acids of the first chain of the protein(Kiefer et al., 2008). Other structures with more sequence coverage in terms of the number of the amino acids than 1K3BA structure were also found, but the X-ray structure of the 1K3BA was selected for two reasons; Firstly, the complete sequence identity with the mutated region, and secondly, its better quality for the purpose of the homology modeling that can exactly describe the structural defects caused by the mutation.

The selected 1K3BA structure was submitted for homology modeling using the online Swiss-Prot server for automated modeling on http://swissmodel.expasy.org(Biasini et al., 2014; Guex \& Peitsch, 1997; Schwede, Kopp, Guex, \& Peitsch, 2003). The result was set for the Energy Minimization job using ZMM software. The ZMM uses the Amber all-atom force field with a cut-off distance of $10 \AA$ and Monte Carlo Minimization Method to minimize conformational energy in the space of generalized coordinates including torsions and bond angles(Zhenqin Li \& Scheraga, 1987; Zdobnov \& Apweiler, 2001). The Energy Minimization was terminated after 100 sequential minimizations failed to improve the lowest-energy conformation.

The essential accuracy and correctness of the model was then evaluated using the PROCHECK and WHATIF programs from the online server at http://nihserver.mbi.ucla.edu/SAVES/(Vriend, 1990).

The electrostatic potential of the molecule was computed using Coulomb's Law and the Swiss-PdbViewer 4.02, as well as the graphical representations presented here(Guex \& Peitsch, 1997).

Results

A six year old boy that was diagnosed as Papillon-Lefevre syndrome affected (according to the established criteria by Gorlin et al.) was referred to Avicenna Research Institute with chief complain of a long history of hyperkeratosis and periodontitis. The majority of his deciduous teeth fell off by the age of 6 years. The family history revealed that he was born from a relative parents and the only affected one of the family(Guex \& Peitsch, 1997; Zhiming Li et al., 2014). His cousin shows clinical symptoms of the disease but his siblings seem normal at the time of study (Fig 1a, 1b and 1c). The complete analysis of coding sequences and splice sites of CTSC gene revealed a novel three nucleotides GAC insertion (p.122insT) in the third exon of the gene in-patient in homozygous form, which lead to the insertion of an additional threonine in polypeptide chain. Further genotype analyses of parents showed the existence of mentioned mutation in heterozygous form (Fig 2). All the healthy control volunteers showed the wild type sequence of the gene.

The tertiary structures of the normal and mutated proteins are shown in Fig. 3. The model was also analyzed in terms of stereo-chemical and geometrical parameters such as G-Factor, bond length, and bond angles, for which the results be satisfied with the discussed criteria. In addition, most of the residues were inside the favorable regions of the Ramachandran map. After energy minimization, the overall energy of the model was $-666.19 \mathrm{kcal} / \mathrm{mol}$ for the $1 \mathrm{~K} 3 \mathrm{BA}$ structure.

Structural changes that have been emerged by the insertion of the Threonine amino acid in the normal gene (p.122ins.T), as predicted from molecular modeling, are compatible with the observed phenotype.

Discussion

Although PLS is a rare disease, its psychological and social impacts on growing affected children can influence the quality of their life. The exact etiology of PLS is still ambiguous. However, Anatomy, microorganisms, immunologic response and genetic factors are responsible for development of the syndrome. Some microbial agents including Actinobacillus actinomycetemcomitans was reported as a significant factor in development of periodontal involvements(Stabholz, Taichman, \& Soskolne, 1995). Recent investigations have demonstrated that PLS is mostly seen in consanguineous marriages, which is directly related to the cathepsin C abnormality. Dipeptidyl peptidase I (DPPI) or cathepsin C has a vital role in defense against pathogenic organisms by physiological activating of some serine proteases in immune cells. Therefore, deficiency of cathepsin C function will be resulted in lack of immunological response, leading to increased risk of severe infections(Basapogu Sreeramulu et al., 2015). The CTSC gene that code cathepsin C, is commonly expressed in epithelial cells 
and causes different clinical symptoms such as severe gingivostomatitis and periodontitis. Early diagnosis of PLS has important role in management of patient's oral conditions.

In this study, we report a novel mutation in CTSC gene, which can lead to production of an abnormal protein according to the obtained results of DNA genomic sequencing and in Silico studies.

Genetic testing of studied family revealed presence of the novel insertion in homozygous form in the third exon of CTSC gene (p.122ins.T) of affected patient. Up till now $85 \%$ of PLS patients are in homozygous form in CTSC mutations which $13 \%$ are located in exon 1 to 3, located in the exclusion domain of the related protein (Nagy et al., 2014). Coding sequence of cathepsin c contains 463 amino acids including a signal peptide with 24 amino acids, an exclusion domain with 233 amino acids and a propeptide with 206 amino acids that it also contains the heavy and light chain regions(Nitta et al., 2005). The C-terminal portion of the exclusion domain is a conserved region, which is thought to be important for enzyme activity. Mutation in this region blocks access to the active site and inhibits enzyme to bind substrates.

Structural analyses of the mutated protein also revealed important changes in its tertiary structure due to the elucidated three nucleotides insertion (p.122ins.T) in the active site of Dipeptidyl peptidase I domain (cathepsin C), exclusion domain, SSF75001, accessed by InterproScan(Zdobnov \& Apweiler, 2001). As in Figure 3a, which is related to the normal protein model, the amino acids threonine, methionine, and threonine are at positions 121, 122, and 123 in exclusion domain of the protein, respectively, but after the mutation (p.122ins.T), a threonine after threonine 121 is added. Proximity of three threonines can also alter the protein structure, leading to poor or inappropriate protein function; this change is shown in figure $3 \mathrm{~b}$. Therefore, due to the strong relationships between the protein tertiary structure and its functions, it can be realized that such important abnormality in the structure of the mutated protein can be cause consequent important abnormalities in the protein function.

In conclusion, according to the results obtained from this study, we can justify correlation between this reported mutation and observed phenotype in this 6-year-old-boy affected by Papillon-Lefevre syndrome.

Acknowledgment

This work was supported by a grant from the Vice Chancellor for Research at Mashhad University of Medical Sciences, no. 910523.

Conflict of interest

The authors declare no potential conflicts of interest.

References

Almuneef, M., Al Khenaizan, S., Al Ajaji, S., \& Al-Anazi, A. (2003). Pyogenic liver abscess and PapillonLefèvre syndrome: not a rare association. Pediatrics , 111 (1), e85-e88.

Arnold, K., Bordoli, L., Kopp, J., \& Schwede, T. (2006). The SWISS-MODEL workspace: a web-based environment for protein structure homology modelling. Bioinformatics , 22 (2), 195-201.

Biasini, M., Bienert, S., Waterhouse, A., Arnold, K., Studer, G., Schmidt, T., . . Bordoli, L. (2014). SWISSMODEL: modelling protein tertiary and quaternary structure using evolutionary information.Nucleic Acids Research , 42 (W1), W252-W258.

Borroni, G., Pagani, A., Carcaterra, A., Pericoli, R., Gabba, P., \& Marconi, M. (1985). Immunological alterations in a case of Papillon-Lefèvre syndrome with recurrent cutaneous infections.Dermatology , 170 (1), 27-30.

Cury, V. F., Costa, J. E., Gomez, R. S., Boson, W. L., Loures, C. G., \& Marco, L. De. (2002). A novel mutation of the cathepsin C gene in Papillon-Lefevre syndrome. Journal of Periodontology ,73 (3), 307-312.

de Haar, S. F., Jansen, D. C., Schoenmaker, T., Vree, H. De, Everts, V., \& Beertsen, W. (2004). Lossof-function mutations in cathepsin $\mathrm{C}$ in two families with Papillon-Lefevre syndrome are associated with 
deficiency of serine proteinases in PMNs. Human Mutation , 23 (5), 524.

Farjadian, S., Kiyanimanesh, N., Abbaszadegan, A., \& Lotfazar, M. (2007). HLA class I gene polymorphism in Iranian patients with Papillon-Lefevre Syndrome. Iranian Journal of Immunology ,4 (4), 241-245.

Farjadian, S., Lotfazar, M., \& Ghaderi, A. (2008). Analysis of human leukocyte antigen class II gene polymorphism in Iranian patients with Papillon-Lefevre syndrome: a family study. Iranian Journal of Immunology , 5 (3), 171-176.

Farkas, K., Paschali, E., Papp, F., Valyi, P., Szell, M., Kemeny, L., .. Csoma, Z. (2013). A novel sevenbase deletion of the CTSC gene identified in a Hungarian family with Papillon-Lefevre syndrome.Archives of Dermatological Research , 305 (5), 453-455.

Gorlin, R J, Sedano, H., \& Anderson, V. E. (1964). The syndrome of palmar-plantar hyperkeratosis and premature periodontal destruction of the teeth: a clinical and genetic analysis of the Papillon-Lefevre syndrome. The Journal of Pediatrics , 65 (6), 895-908.

Gorlin, Robert J. (2000). Of palms, soles, and gums. Journal of Medical Genetics, 37 (2), 81-82.

Gorlin, Robert J, Cohen Jr, M. M., \& Hennekam, R. C. M. (2001). Syndromes of the head and neck. Oxford University Press.

Guex, N., \& Peitsch, M. C. (1997). SWISS-MODEL and the Swiss-Pdb Viewer: an environment for comparative protein modeling.Electrophoresis , 18 (15), 2714-2723.

Haneke, E. (1979). The Papillon-Lefevre syndrome: keratosis palmoplantaris with periodontopathy. Human Genetics ,51 (1), 1-35.

Hart, T. C., Hart, P. S., Bowden, D. W., Michalec, M. D., Callison, S. A., Walker, S. J., .. Firatli, E. (1999). Mutations of the cathepsin C gene are responsible for Papillon-Lefevre syndrome. Journal of Medical Genetics , 36 (12), 881-887.

http://www.hgmd.cf.ac.uk/ac/gene.php?gene=CTSC . (n.d.). http://www.hgmd.cf.ac.uk/ac/gene.php?gene=CTSC.

Idon, P., Olasoji, H., \& Fusami, M. (2015). Papillon-Lefevre syndrome: review of literature and report of three cases in the same family. The Nigerian Postgraduate Medical Journal, 22 (1).

Kanthimathinathan, H. K., Browne, F., Ramirez, R., McKaig, S., Debelle, G., Martin, J., .. Moss, C. (2013). Multiple cerebral abscesses in Papillon-Lefevre syndrome. Child's Nervous System , 29 (8), 12271229 .

Khandpur, S., \& Reddy, B. S. N. (2001). Papillon-Lefevre syndrome with pyogenic hepatic abscess: a rare association. Pediatric Dermatology , 18 (1), 45-47.

Kiefer, F., Arnold, K., Kunzli, M., Bordoli, L., \& Schwede, T. (2008). The SWISS-MODEL Repository and associated resources. Nucleic Acids Research , 37 (suppl_1), D387-D392.

Li, Zhenqin, \& Scheraga, H. A. (1987). Monte Carlo-minimization approach to the multiple-minima problem in protein folding. Proceedings of the National Academy of Sciences , 84 (19), 6611-6615.

Li, Zhiming, Liu, J., Fang, S., Zhu, H., Zhang, X., Cai, J., .. Xu, Y. (2014). Novel compound heterozygous mutations in CTSC gene cause Papillon-Lefevre syndrome with high serum immunoglobulin E. Journal of Dermatological Science, 76 (3), 258-260.

Lundgren, T., Crossner, C., Twetman, S., \& Ullbro, C. (1996). Systemic retinoid medication and periodontal health in patients with Papillon-Lefevre syndrome. Journal of Clinical Periodontology ,23 (3), 176-179.

Lundgren, T., \& Renvert, S. (2004). Periodontal treatment of patients with Papillon-Lefevre syndrome: a 3-year follow-up. Journal of Clinical Periodontology, 31 (11), 933-938. 
Mercy, P., Singh, A., Ghorpade, A. K., Das, M., \& Upadhyay, A. (2013). Papillon-Lefevre syndrome: two siblings, one developing liver abscess.Indian J Dermatol , 58 (5), 410.

Moghaddasian, M., Arab, H., Dadkhah, E., Boostani, H., Babak, A. R., \& Abbaszadegan, M. R. (2014). Protein modeling of cathepsin C mutations found in Papillon-Lefevre syndrome. Gene, 538 (1), 182-187.

Morgan, R. D., Hannon, E., \& Lakhoo, K. (2011). Renal abscess in Papillion-Lefevre syndrome. Pediatric Surgery International ,27 (12), 1381-1383.

Nagy, N., Valyi, P., Csoma, Z., Sulak, A., Tripolszki, K., Farkas, K., .. Fabos, B. (2014). CTSC and Papillon-Lefevre syndrome: detection of recurrent mutations in $\mathrm{H}$ ungarian patients, a review of published variants and database update. Molecular Genetics $\&$ Genomic Medicine , 2 (3), 217-228.

Nitta, H., Wara-aswapati, N., Lertsirivorakul, J., Nakamura, T., Yamamoto, M., Izumi, Y., ... Ishikawa, I. (2005). A Novel Mutation of the Cathepsin C Gene in a Thai Family With Papillon-Lefevre Syndrome. Journal of Periodontology , 76 (3), 492-496.

Oğuzkurt, P., Tanyel, F. C., Büyükpamukçu, N., \& Hiçsönmez, A. (1996). Increased risk of pyogenic liver abscess in children with Papillon-Lefevre syndrome. Journal of Pediatric Surgery, 31 (7), 955-956.

Pallos, D., Acevedo, A. C., Mestrinho, H. D., Cordeiro, I., Hart, T. C., \& Hart, P. S. (2010). Novel cathepsin $\mathrm{C}$ mutation in a Brazilian family with Papillon-Lefèvre syndrome: case report and mutation update.Journal of Dentistry for Children, 77 (1), 36-41.

Papillon, M. (1924). Deux cas de keratodernune palmaire symmetrique famiale (Maladie de Meleda) chez Le frere et la soeur. Coexistece dans les deux cas d'alterations dentaires graves. BUll Soc Fr Dermatol Syp Lt , $31,82-84$.

Romero-Quintana, J. G., Frías-Castro, L. O., Arámbula-Meraz, E., Aguilar-Medina, M., Dueñas-Arias, J. E., Melchor-Soto, J. D., ... Ramos-Payán, R. (2013). Identification of novel mutation in cathepsin C gene causing Papillon-Lefèvre Syndrome in Mexican patients. BMC Medical Genetics , 14 (1), 7.

Schwede, T., Kopp, J., Guex, N., \& Peitsch, M. C. (2003). SWISS-MODEL: an automated protein homologymodeling server. Nucleic Acids Research, 31 (13), 3381-3385.

Sreeramulu, B, Haragopal, S., Shalini, K., Sudha, M. D., \& Kiran, G. (2012). The prosthodontic management of a young edentulous patient with the papillon lefevre syndrome-a rare case report. Journal of Clinical and Diagnostic Research: JCDR , 6 (10), 1808.

Sreeramulu, Basapogu, Shyam, N. D. V. N., Ajay, P., \& Suman, P. (2015). Papillon-Lefèvre syndrome: clinical presentation and management options. Clinical, Cosmetic and Investigational Dentistry ,7 , 75.

Stabholz, A., Taichman, N. S., \& Soskolne, W. A. (1995). Occurrence of Actinobacillus actinomycetemcomitans and anti-leukotoxin antibodies in some members of an extended family affected by Papillon-Lefèvre syndrome. Journal of Periodontology , 66 (7), 653-657.

Tekin, B., Yucelten, D., Beleggia, F., Sarig, O., \& Sprecher, E. (2016). Papillon-Lefèvre syndrome: report of six patients and identification of a novel mutation. International Journal of Dermatology , 55 (8), 898-902.

Toomes, C., James, J., Wood, A. J., Wu, C. L., McCormick, D., Lench, N., .. Woods, C. G. (1999). Loss-offunction mutations in the cathepsin $\mathrm{C}$ gene result in periodontal disease and palmoplantar keratosis. Nature Genetics, 23 (4), 421.

Tosti, A., Manuzzi, P., Bardazzi, F., \& Costa, A. (1988). Is etretinate dangerous in Papillon-Lefèvre syndrome? Dermatology ,176 (3), 148-150.

Vriend, G. (1990). WHAT IF: a molecular modeling and drug design program. Journal of Molecular Graphics , $8(1), 52-56$. 
Wani, A. A., Devkar, N., Patole, M. S., \& Shouche, Y. S. (2006). Description of two new cathepsin C gene mutations in patients with Papillon-Lefevre syndrome. Journal of Periodontology ,77 (2), 233-237.

Wu, W., Chen, B., Chen, X., Chen, L., Yi, L., Wang, Y., ... Sun, W. (2016). A novel large deletion combined with a nonsense mutation in a $\mathrm{C}$ hinese child with $\mathrm{P}$ apillon-L efevre syndrome. Journal of Periodontal Research , 51 (3), 376-380.

Zdobnov, E. M., \& Apweiler, R. (2001). InterProScan-an integration platform for the signature-recognition methods in InterPro.Bioinformatics , 17 (9), 847-848.

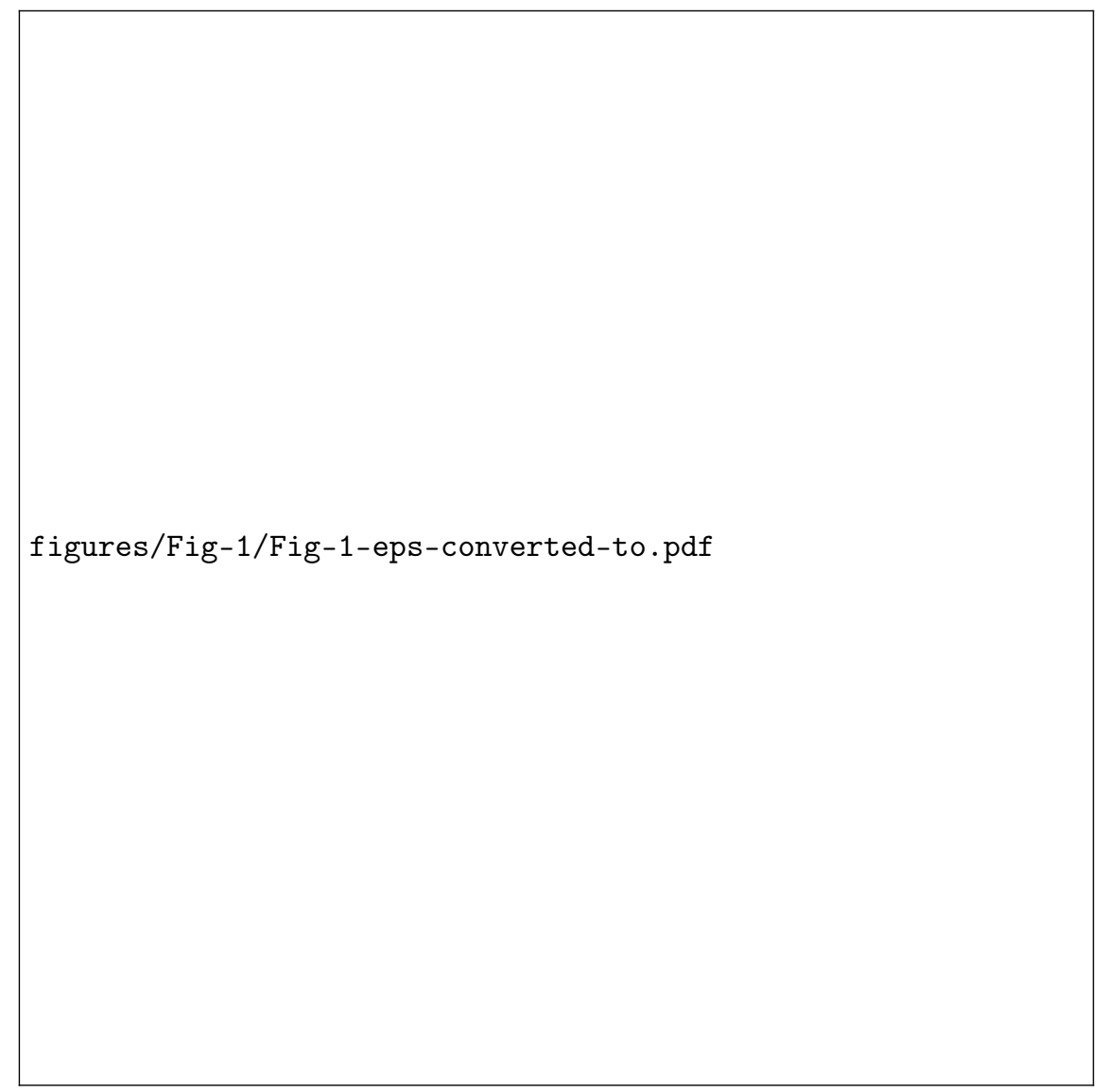


figures/Fig-2/Fig-2-eps-converted-to.pdf 
figures/Fig-3/Fig-3-eps-converted-to.pdf 